\title{
Murphy, Kim: Government communications in a digital age. A comparative study of online government communications in Germany and Great Britain
}

\author{
Baden-Baden: Nomos 2019. 276 Seiten. Preis: $€ 54$
}

\section{Dennis Lichtenstein}

Online publiziert: 12 . Juni 2020

(C) The Editors of the Journal 2020

In der aktuellen COVID-19 Krise zitiert nicht nur die Tagesschau regelmäßig aus dem Kanzlerin-Podcast. Auch auf Social Media diffundieren Botschaften der Bundesregierung, die über das Virus aufklären, Krisenpolitik begründen und an die Bürger_innen appellieren. Regierungskommunikation, so scheint es, hat die digitalen Kanäle für sich entdeckt! Die Dissertation von Kim Murphy, die sie im Rahmen der Forschergruppe Politische Kommunikation in der Onlinewelt verfasst und an der FU Berlin eingereicht hat, untersucht, wie Regierungsorganisationen Onlineplattformen nutzen, um mit Bürgern_innen und Medien zu kommunizieren. Sie adressiert damit ein aktuelles und bislang zu wenig beachtetes Thema. Die Schrift ergänzt bisherige Arbeiten zur Anpassung etablierter Akteure an die neuen Bedingungen der Onlinewelt, die sich vor allem mit Parteien in Wahlkampfkontexten und mit klassischen Nachrichtenmedien befasst haben.

Kim Murphy verbindet eine organisationstheoretische Perspektive mit Überlegungen zu den Logiken digitaler Netzwerke, die Kommunikationsstrukturen und Interaktionen entscheidend verändern. Wie sich die Büros von Regierungschefs, Ministerien und die institutionalisierte Öffentlichkeitsarbeit an neue Erfolgsfaktoren wie Individualisierung und Vernetzung anpassen, wird demnach von gewachsenen Organisationsstrukturen, Zielen und Ressourcen sowie von den Rahmenbedingungen medialer und politischer Systeme angeleitet. In einem umfassenden Überblick zum Forschungsstand arbeitet Kim Murphy bezogen auf ihre Untersuchungsländer, Deutschland und Großbritannien, Einflussfaktoren auf der Makro- und Mesoebene heraus. Sie kritisiert Modelle der komparativen Forschung, die der Organisationsebene bislang wenig Beachtung geschenkt haben, und zeigt, wie die Netzwerklogik klassische Ebenenunterscheidungen unterwandert.

D. Lichtenstein $(\triangle)$

Österreichische Akademie der Wissenschaften, Vordere Zollamtsstr. 3, 1030 Wien, Österreich

E-Mail: Dennis.Lichtenstein@oeaw.ac.at 
In der empirischen Untersuchung möchte Kim Murphy klären, 1) wie intensiv und auf welche Weise deutsche und britische Regierungsorganisationen Websites und Social Media zur Kommunikation mit Bürger_innen und Medien nutzen und 2) wie sich Regierungsorganisationen untereinander und mit anderen Akteuren vernetzen. Kernstück der Studie ist eine systematische Inhaltsanalyse der Websites und Social Media-Auftritte von insgesamt 47 nationalen Regierungsorganisationen auf Facebook, Twitter und YouTube. Ergänzend wird eine explorative Social Network Analysis zu den Interaktionen der Organisationen auf Twitter durchgeführt. Die Studie untersucht einen Ausschnitt von zwei Wochen politischer Routinekommunikation im Mai und Juni 2015. Sie liefert damit keine brandaktuellen Einblicke, sondern bezieht sich auf die Regierungen von Merkel/Gabriel in Deutschland (2013-2018) und Cameron (2015-2016) in Großbritannien.

Die Befunde bestätigen zu großen Teilen die aus dem Forschungsstand abgeleiteten Überlegungen. Sie zeigen, dass die Regierungskommunikation den digitalen Raum erreicht hat, dort aber längst nicht alle interaktiven Potenziale der Netzwerklogik ausschöpft. Regierungskommunikation online verläuft in allererster Linie als Selbstdarstellung und Informationsvermittlung Top-Down. Websites und Twitter sind die zentralen Kanäle. Interaktionen oder eine Ermutigung der Bürger_innen zur Partizipation finden über die untersuchten Plattformen hinweg kaum statt. Länderunterschiede zeigen sich mit Blick auf die in Deutschland stark ausgeprägte Transparenz zur Verantwortlichkeit für Onlineinhalte. Auf Twitter bilden die Retweets der Organisationen in Großbritannien anders als in Deutschland ein dichtes Netzwerk ab, das zu Medien und anderen Akteuren kaum geöffnet ist. Hierin spiegelt sich der hohe Institutionalisierungsgrad britischer Regierungskommunikation wider.

Innerhalb der Länder zeigen sich interessante Unterschiede zwischen einzelnen Organisationen und Akteuren. Es sind die weniger profilierten Minister_innen und Ministerien, die den Schritt in die Interaktion wagen und dadurch Netzwerkstrukturen stärken. Das geschieht auch unter Nutzung persönlicher Social Media-Seiten, die dem Anspruch der Netzwerklogik auf Individualisierung entsprechen, aber Rollengrenzen zwischen Regierungs- und Parteiidentität aufweichen. Als early adopters geben diese Akteure Hinweise auf die künftige Entwicklung der Regierungskommunikation online und werfen zugleich ethische und rechtliche Fragen nach Transparenz und der Verwendung von Regierungsressourcen auf.

Eine systematische Analyse von Einflussfaktoren auf der Makro- und Mesoebene auf die Regierungskommunikation etwa über eine Regressionsanalyse kann die Arbeit nicht leisten. Der Abgleich findet im Rahmen einer Diskussion statt, die stellenweise spekulativ ausfällt, aber die Befunde größtenteils überzeugend in den Forschungsstand einordnet. Die Arbeit sagt auch nichts zu den Inhalten der Regierungskommunikation aus und kann damit nicht klären, inwieweit sich auffällige Aktivitäten einzelner Organisationen über aktuelle politische Projekte erklären lassen. Zudem bleiben normative Überlegungen zur Informationsvermittlung und Deliberation mit den Bürger_innen in der Diskussion der Befunde weitestgehend ausgespart. Für die weitere Forschung zu diesen und ähnlichen Aspekten leistet die Studie aber eine wichtige Bestandsaufnahme und gibt auch theoretisch interessierten Leser_innen vielversprechende Impulse. Die Dissertation folgt einem klaren roten Faden und zur Orientierung trägt nicht zuletzt die Vielzahl an Tabellen bei, die 
Schlüsselinformationen zu Theorie, Methode und zu den Befunden systematisiert zusammenfassen.

PD Dr. Dennis Lichtenstein ist Postdoktorand am Institut für vergleichende Medien- und Kommunikationsforschung an der Österreichischen Akademie der Wissenschaften in Wien und der Universität Klagenfurt. 